\title{
Quality of Minimal Sets of Prime Implicants of Boolean Functions
}

\author{
V. C. Prasad
}

\begin{abstract}
Two new problems are posed and solved concerning minimal sets of prime implicants of Boolean functions. It is well known that the prime implicant set of a Boolean function should be minimal and have as few literals as possible. But it is not well known that min term repetitions should also be as few as possible to reduce power consumption. Determination of minimal sets of prime implicants is a well known problem. But nothing is known on the least number of (i) prime implicants (ii) literals and (iii) min term repetitions, any minimal set of prime implicants will have. These measures are useful to assess the quality of a minimal set. They are then extended to determine least number of prime implicants / implicates required to design a static hazard free circuit. The new technique tends to give smallest set of prime implicants for various objectives.
\end{abstract}

Keywords - Logic circuits, Digital circuits, Boolean functions, Minimal sets

\section{INTRODUCTION}

$\mathrm{D}_{\mathrm{a}}$ ETERMINATION of a minimal set of prime implicants of a Boolean function is a fundamental problem in combinational logic circuit design $[1-4,6]$. This is required to reduce the size of the logic circuit. Given two such minimal sets with same number of prime implicants, we would like to use the one with less number of literals $\left[\begin{array}{ll}1 & -3\end{array}\right]$. This is done primarily to reduce the number of inputs (fan in) of gates. Thus minimal sets of prime implicants and literals are important in logic circuit design. This attracted the attention of a large number of researchers in the last eighty years. In spite of that there are some important gaps in the literature. This paper points out two such gaps and provides answers to them. The first one concerns the nature of a minimal set. The other concerns the least number of prime implicants any minimal set will have.

It is common practice to include a min term in as many prime implicants as possible. This is done to reduce the number of literals of an implicant as much as possible. Consequently some min terms are covered more than once in a minimal set of prime implicants. But these repetitions must be avoided as far as possible to reduce power consumption. Therefore given two minimal sets with same number of prime implicants, we should choose the set with lesser repetitions of min terms. Thus the best minimal set of prime implicants will have least number of (i) prime implicants (ii) literals (iii) min term repetitions. Calculation of minimal sets of prime implicants is extensively discussed in the literature and it is widely taught in undergraduate courses of many disciplines of engineering. But there are no measures in the literature to know how "good" is the set so calculated. This paper fills that

V. C. Prasad is with the Dayalbagh Educational Institute, Dayalbagh, India (Retired from Indian Institute of Technology, Delhi (e-mail: prasadvishdelhi@gmail.com). gap. It presents methods to compute these numbers. In a given situation if a minimal set has numbers close to them, it implies that the minimal set is fairly good. A minimal set derived using the new technique tends to be the smallest for the intended purpose.

A minimal set of prime implicants covers all min terms of a Boolean function. This is not enough to make a logic circuit free of static hazards [1, 2 ]. Additional prime implicants are required to achieve this. Methods are present in the literature to determine these additional prime implicants. But there are no measures to assess the quality of the set. It is shown in this paper that the technique used for normal Boolean functions can be used for hazard free circuits also with a simple modification. Thus it is possible to compute the least number of prime implicants, literals etc required for static hazard free circuits.

Minimal min term set is explained in Section II. Methods to compute the lower bounds mentioned above are developed in Section III. Static hazard free circuits are discussed in Section IV. The contributions of the paper are summarized in Section $\mathrm{V}$.

\section{Minimal Min Term Set}

\section{A. Definitions and Notations}

A min term is a product (AND) term in which every Boolean variable appears in complemented or uncomplemented form. An implicant is a product term of variables of a Boolean function. An implicant is a prime implicant if it does not subsume any other implicant with fewer literals [1]. Two min terms of a Boolean function of $\mathrm{n}$ variables are said to form a dyad if there is an implicant of (n-1) variables covering both of them.

We are given a Boolean function $f$ in the sum (OR) of products (SOP) form. It may or may not be a SOP of min terms. Using this SOP all prime implicants of the function can be determined [ $1,2,3]$. Henceforth we assume that all prime implicants are known. A set of prime implicants is said to be minimal if (i) it covers all min terms of $f$, (ii) no subset of it can do this[1,2,3]. A literal $\mathrm{x}$ of a product term $\mathrm{xY}$ of $f$ can be deleted if $f$ contains $\mathrm{x}^{t} \mathrm{Y}$ also. i.e., $\mathrm{x}$ is redundant in $\mathrm{XY}$. A SOP of $f$ is a minimal literal set if (i) it covers all min terms of $f$ (ii) a subset of product terms does not cover all min terms (iii) no literal is redundant. It is clear from this definition that a minimal literal set is a minimal set of prime implicants also. Similarly a minimal prime implicant set is a minimal literal set also. But if we are given two minimal sets of prime implicants of the function with same number of prime implicants we wish to choose the set with fewer literals because this reduces the fan in of gates. 


\section{B. Minimal Min Term Set}

Deletion of a min term from a product term splits the product term into two or more product terms. For example let $f$ be a Boolean function of four variables A, B, C, D and let $\mathrm{AB}$ be a product term in a SOP of $f$. Then if the min term ABCD is deleted from $\mathrm{AB}$ it can be written as $\mathrm{ABC}^{t}+\mathrm{ABD}^{x}$. But if $\mathrm{ABC}^{t}$ or $\mathrm{ABD}^{t}$ is redundant then $\mathrm{AB}$ can be replaced by just one product term after $\mathrm{ABCD}$ is deleted. Therefore deletion of a min term from a product term P of a SOP is allowed only if (i) it is covered by at least two product terms (ii) $\mathrm{P}$ can be replaced by one product term only. In this sense a min term is redundant in $\mathrm{P}$ if it can be deleted. A SOP of $f$ is a minimal min term set if (i) it covers all min terms of the function (ii) no product term has redundant min terms. Note that a minimal min term set need not be a minimal set of prime implicants. For example let $f=$ $\mathrm{AB}+\mathrm{A}^{y} \mathrm{CD}+\mathrm{AB}^{t} \mathrm{CD}$. The product terms in it constitute a minimal min term set but not a minimal set of prime implicants. But this function can also be written as $f=\mathrm{AB}+\mathrm{CD}$. ( $\mathrm{AB}$, $\mathrm{CD}$ ) is a minimal prime implicant set. It is a minimal min term set also. A minimal prime implicant set may not be a minimal min term set. For example let $f=\mathrm{AB}+\mathrm{BCD}$. ( $\mathrm{AB}, \mathrm{BCD})$ is a minimal set of prime implicants. But it is not a minimal min term set $\left(A B, A^{\prime} B C D\right)$ is a minimal min term set. In fact this function has no minimal prime implicant set which can be a minimal min term set also. In such situations a minimal prime implicant set can be transformed into a minimal min term set by eliminating redundant min terms as explained above.

Consider any logic realization of a SOP in which a min term $\mathrm{x}$ covered by two product terms $\mathrm{P}_{1}$ and $\mathrm{P}_{2}$ has two paths to output when $\mathrm{x}=1$. One of them passes through $\mathrm{P}_{1}$ and the other passes through P 2. AND, OR, NOT and NAND realizations are two well known examples of this kind. In such realizations the load current on inputs increases when $\mathrm{x}=1$ because of multiple paths. Devices and resistors on these paths consume power and dissipate as heat. This is reduced if min term repetitions are as few as possible. This aspect of min terms does not seem to have attracted the attention of researchers all these years.

Sum of product form of all min terms is a minimal min term set. But it requires too many gates. Thus we want a SOP to be a minimal prime implicant set also. In view of this henceforth we will restrict our attention to minimal prime implicant sets with as few min term repetitions as possible.

\section{MEASURES OF Minimal PRIME IMPLICANT SETS}

In this section methods are presented to compute the least number of prime implicants, literals and min term repetitions, any minimal set of prime implicants will have.

\section{A. Least Number of Prime Implicants}

We will determine the least number of prime implicants any minimal set will have. This is done using the concept of distant pair of min terms. A pair of min terms $\left(\mathrm{m}_{\mathrm{i}}, \mathrm{m}_{\mathrm{j}}\right)$ of $f$ is said to be a close pair if there is an implicant covering both of them . Otherwise it is a distant pair. This implies that every close pair lies in at least one prime implicant. Note that a dyad is a close pair. But a close pair may or may not be a dyad. Given $f$ determine all close and distant pairs of min terms. A group of min terms can form a close (distant) set if every pair of min terms in it is a close (distant) pair. A close (distant) set of min terms is maximal if no min term can be added to it without violating the close (distant) pair property. All maximal close (distant) sets can be obtained by merging close (distant) pairs [ 1,2]. Maximal close (distant) sets can also be obtained by dividing the universal set of all min terms of $f$ using all distant ( close) pairs [5]. Let $\mathrm{S}_{\mathrm{d}}=\left(\mathrm{m}_{1}, \mathrm{~m}_{2}, \ldots, \mathrm{m}_{\mathrm{d}}\right)$ be one of the largest distant sets. Since every pair of min terms in it is a distant pair we need $d$ prime implicants to cover them. They may not cover all min terms. Therefore additional prime implicants may be required. This proves the following theorem.

Theorem 1 : Every minimal set of prime implicants of $f$ has at least $\left|S_{d}\right|$ number of prime implicants where $S_{d}$ is the largest distant set and $\left|S_{\mathrm{d}}\right|$ denotes the size $d$ of $\mathrm{S}_{\mathrm{d}}$.

Example 1 : Consider the Boolean function [1]

$f(\mathrm{~A}, \mathrm{~B}, \mathrm{C}, \mathrm{D}, \mathrm{E})=\mathrm{m}_{4}+\mathrm{m}_{5}+\mathrm{m}_{9}+\mathrm{m}_{11}+\mathrm{m}_{12}+\mathrm{m}_{14}$ $+\mathrm{m}_{15}+\mathrm{m}_{27}+\mathrm{m}_{30} \cdot \mathrm{m}_{1}, \mathrm{~m}_{17}, \mathrm{~m}_{25}, \mathrm{~m}_{26}$ and $\mathrm{m}_{31}$ are don't care min terms. $\mathrm{m}_{4}$ denotes $\mathrm{A}^{\prime} \mathrm{B}^{\prime} \mathrm{CD}^{\prime} \mathrm{E}^{\prime}$. Other min terms are also denoted similarly. The prime implicants of the function are [1] $\quad \mathrm{P}_{1}: \mathrm{C}^{t} \mathrm{D}^{t} \mathrm{E}\left(\mathrm{m}_{9}\right), \mathrm{P}_{2}: \mathrm{B} \mathrm{C}^{t} \mathrm{E}\left(\mathrm{m}_{9}, \mathrm{~m}_{11}, \mathrm{~m}\right.$ $\left.{ }_{27}\right), \mathrm{P}_{9}: \mathrm{B} \mathrm{DE}\left(\mathrm{m}_{11}, \mathrm{~m}_{15}, \mathrm{~m}_{27}\right), \mathrm{P}_{4}: \mathrm{B} \mathrm{CD}\left(\mathrm{m}_{14}, \mathrm{~m}_{15}, \mathrm{~m}_{30}\right.$ ), $\mathrm{P}_{5}: \mathrm{ABD}\left(\mathrm{m}_{27}, \quad \mathrm{~m}_{30}\right), \mathrm{P}_{6}: A^{t} \mathrm{~B}^{t} \mathrm{D}^{t} \mathrm{E}\left(\mathrm{m}_{5}\right)$,

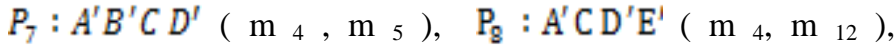
$P_{9}: A^{y} B C E^{t}\left(\mathrm{~m}_{12}, \mathrm{~m}_{14}\right)$. The min terms they cover are shown in brackets. Distant min term pairs are easily determined from this information. For example consider $\mathrm{m}_{4} . \mathrm{m}_{4}$ lies in $\mathrm{P}$ ${ }_{7}$ and $\mathrm{P}_{8}$. Therefore all min term pairs of $\mathrm{m}_{4}$ except those of $\mathrm{P}_{7}$ and $\mathrm{P}_{8}$ are distant pairs. Thus distant min term pairs of $\mathrm{m}$ 4 are $\left(\mathrm{m}_{4}, \mathrm{~m}_{9}\right),\left(\mathrm{m}_{4}, \mathrm{~m}_{11}\right),\left(\mathrm{m}_{4}, \mathrm{~m}_{14}\right),\left(\mathrm{m}_{4}, \mathrm{~m}_{15}\right)$, ( $\left.\mathrm{m}_{4}, \mathrm{~m}_{27}\right)$ and $\left(\mathrm{m}_{4}, \mathrm{~m}_{30}\right)$. Distant pairs of other min terms can also be determined similarly. $\mathrm{U}=\left\{\mathrm{m}_{4}, \mathrm{~m}_{5}, \mathrm{~m}_{9}, \mathrm{~m}_{11}, \mathrm{~m}\right.$ $\left.12, \mathrm{~m}_{14}, \mathrm{~m}_{15}, \mathrm{~m}_{27}, \mathrm{~m}_{30}\right\}$ is the set of all min terms excluding don't cares. Since every pair of min terms of a prime implicant is a close pair, retain only one min term of a prime implicant and delete others. Do this in all possible ways and for all prime implicants. This divides $U$ into many subsets. Eliminate a set if it is a subset of another set. At the end of this we will have all maximal distant sets. Applying this to U using $\mathrm{P}_{1}, \mathrm{U}$ is not further subdivided because $\mathrm{P}_{1}$ has one min term only . $\mathrm{P}_{2}$ has three min terms $\left(\mathrm{m}_{9}, \mathrm{~m}_{11}, \mathrm{~m}_{27}\right) .\left(\mathrm{m}_{9}, \mathrm{~m}_{11}\right)$, $\left(\mathrm{m}_{11}, \mathrm{~m}_{27}\right)$ and $\left(\mathrm{m}_{9}, \mathrm{~m}_{27}\right)$ are three possible close pairs of min terms which must be deleted from $U$ to destroy all close pairs of $\mathrm{P}_{2}$.This divides $\mathrm{U}$ into three sets .They are $\left(\mathrm{m}_{4}, \mathrm{~m}\right.$ $\left.5, \mathrm{~m}_{12}, \mathrm{~m}_{14}, \mathrm{~m}_{15}, \mathrm{~m}_{27}, \quad \mathrm{~m}_{30}\right),\left(\mathrm{m}_{4}, \mathrm{~m}_{5}, \mathrm{~m}_{9}, \mathrm{~m}_{12}, \mathrm{~m}\right.$ $\left.{ }_{14}, \mathrm{~m}_{15}, \mathrm{~m}_{30}\right),\left(\mathrm{m}_{4}, \mathrm{~m}_{5}, \mathrm{~m}_{11}, \mathrm{~m}_{12}, \mathrm{~m}_{14}, \mathrm{~m}_{15}, \mathrm{~m}_{30}\right)$. To destroy close pairs of $\mathrm{P}_{3}$, delete $\left(\mathrm{m}_{11}, \mathrm{~m}_{15}\right),\left(\mathrm{m}_{15}, \mathrm{~m}_{27}\right.$ ) and $\left(\mathrm{m}_{11}, \mathrm{~m}_{27}\right)$ from each of these sets. Do like this to all prime implicants. At the end we get all maximal distant sets. ( $\left.\mathrm{m}_{5}, \mathrm{~m}_{9}, \mathrm{~m}_{12}, \mathrm{~m}_{30}\right),\left(\mathrm{m}_{5}, \mathrm{~m}_{11}, \mathrm{~m}_{12}, \mathrm{~m}_{30}\right),\left(\mathrm{m}_{5}, \mathrm{~m}_{9}\right.$, $\left.\mathrm{m}_{12}, \mathrm{~m}_{15}\right)$ etc are some of the largest distant sets . According to Th. 1 this implies that every minimal set of prime implicants covering all min terms of this function will have at least four prime implicants. All minimal sets are easily obtained [1] using Petrick's method revealing that this statement is true.

Consider the largest distant set $\left(\mathrm{m}_{5}, \mathrm{~m}_{9}, \mathrm{~m}_{12}, \mathrm{~m}_{30}\right) . \mathrm{P}_{6}$ with one min term and $\mathrm{P}_{7}$ with two min terms cover $\mathrm{m}_{5}$. Choose $\mathrm{P}_{7}$ to cover $\mathrm{m}_{5}$ because it covers more min terms. Similarly choose $\mathrm{P}_{2}, \mathrm{P}_{9}$ and $\mathrm{P}_{4}$ to cover $\mathrm{m}_{9}, \mathrm{~m}_{12}$ and $\mathrm{m}_{30}$ respectively. The set $\left(\mathrm{P}_{7}, \mathrm{P}_{2}, \mathrm{P}_{9}, \mathrm{P}_{4}\right)$ covers all min 
terms . Therefore this set is the smallest minimal set of prime implicants of $f$. This shows that if prime implicants covering min terms of largest distant set are selected carefully then we can get the smallest minimal set.

Remarks:

1. If we apply this approach to min terms of $\bar{f}(f=0)$, we get the least number of prime implicates which any POS ( product of sums) of $f$ must have. We can realize $f$ using prime implicants of $\bar{f}$ and an inverter at the output. In this sense the least number of prime implicants we need to realize $f$ equals the smaller of $\left|S_{\mathrm{d}}\right|$ and $\left|\overline{S_{\mathrm{d}}}\right|$ where $\left|\overline{S_{\mathrm{d}}}\right|$ denotes the size of the largest distant set of $\bar{f}$.

2. Min terms of don't care conditions are not included in this calculation.

3. We can extend this to multiple Boolean functions as follows: Obtain all distant pairs of each function. $\left(\mathrm{m}_{\mathrm{i}}, \mathrm{m}_{\mathrm{j}}\right)$ is neither a close pair nor a distant pair if $\mathrm{m}_{\mathrm{i}}$ and $\mathrm{m}_{\mathrm{j}}$ belong to different functions. If a pair is a distant pair for one function and a close pair for another function then take it as a distant pair. Using the pairs so obtained determine the largest distant set $S_{d}$. $\left|S_{d}\right|$ gives the least number of prime implicants required to cover min terms of all functions.

4. We don't have to know all prime implicants to use the concepts of close and distant pairs. Let $U_{p}$ be the set of all prime implicants and $S_{p}$ be a subset of $U_{p}$. We can define close and distant pairs with respect to $\mathrm{S}_{\mathrm{p}}$ also. A close (distant) pair with respect to $U_{p}\left(S_{p}\right)$ may or may not be a close (distant) pair with respect to $S_{p}\left(U_{p}\right)$. Therefore least number of prime implicants with respect to $S_{p}$ can be more.

5. Compatible and incompatible pairs of states are well known concepts in Sequential circuits. They are used in sequential circuit design $[1,2,5]$. The concept of compatibility occurs naturally in the state tables of sequential circuits. It depends upon next states and outputs for all inputs. But there is no equivalent concept for min terms in the truth tables of Boolean functions. Close and distant pairs of min terms depend on implicants and hence prime implicants and not truth tables. So close and distant pairs can change with prime implicants sets. But compatible and incompatible states are fixed for a given state table.

\section{B. Least Number of Literals}

Let $\mathrm{S}_{\mathrm{d}}=\left(\mathrm{m}_{1}, \mathrm{~m}_{2}, \ldots, \mathrm{m}_{\mathrm{d}}\right)$ be the largest distant set of min terms. Let $\mathrm{P}_{k}$ be a prime implicant with least number of literals covering the min term $\mathrm{m}_{\mathrm{k}}$ for all $\mathrm{k}=1,2, \ldots, \mathrm{d}$. Then $\mathrm{S}_{\mathrm{pd}}=\left(\mathrm{P}_{1}, \mathrm{P}_{2}, \ldots, \mathrm{P}_{\mathrm{d}}\right)$ is a set of prime implicants covering all min terms of $\mathrm{S}_{\mathrm{d}}$. Let $l$ be the total number of literals of the prime implicants of $\mathrm{S}_{\mathrm{pd}}$.

Theorem 2: Any minimal set of prime implicants of $f$ will have at least $l$ literals.

Proof: Let $\mathrm{X}=\left\{\mathrm{P}_{\mathrm{x} 1}, \mathrm{P}_{\mathrm{x} 2}, \ldots, \mathrm{P}_{\mathrm{xb}}\right\}$ be a minimal set of prime implicants covering all min terms of $f$ such that the total number of literals in $\mathrm{X}$ is the lowest. $\mathrm{X}$ covers the min terms of $\mathrm{S}_{\mathrm{d}}$ also. Therefore from Th. $1 \mathrm{~b} \geq \mathrm{d}$. Let $\mathrm{P}_{\mathrm{xk}}$ be the prime implicant of $\mathrm{X}$ with least number of literals covering $\mathrm{m}$
${ }_{k}$ of $S_{d}$ for all $k=1,2, \ldots, d$. But $P_{k}$ of $S_{p d}$ also covers $m_{k}$. Since $\mathrm{P}_{\mathrm{k}}$ has least literals $\left|\mathrm{P}_{\mathrm{K}}\right| \geq\left|\mathrm{P}_{\mathrm{k}}\right|$ where $|\mathrm{P}|$ denotes the number of literals in the product term P. Since every pair of min terms of $S_{d}$ is a distant pair, $P_{x k}$ that covers min term $\mathrm{m}$ ${ }_{k}$ of $\mathrm{S}_{\mathrm{d}}$ cannot cover other min terms of $\mathrm{S}_{\mathrm{d}}$.

$$
\begin{aligned}
& \ldots\left|\mathrm{P}_{\mathrm{x} 1}\right|+\left|\mathrm{P}_{\mathrm{x} 2}\right|+\ldots .+\left|\mathrm{P}_{\mathrm{xd}}\right| \geq\left|\mathrm{P}_{1}\right|+\left|\mathrm{P}_{2}\right|+ \\
& \ldots .+\left|\mathrm{P}_{\mathrm{d}}\right|=l \\
& +\left|\mathrm{P}_{\mathrm{xd}}\right|+\left|\mathrm{P}_{\mathrm{x}} \mathrm{d}+1\right|+\ldots \ldots+\left|\mathrm{P}_{\mathrm{x} 1}\right|+\left|\mathrm{P}_{\mathrm{x} 2}\right|+\ldots \\
& \text { i.e., } \\
& \text { result. }
\end{aligned}
$$

A minimal set of prime implicants with as few literals as possible can be obtained as follows : Choose a prime implicant with least number of literals to cover a min term not covered so far. Do this for all min terms to get an ordered set of prime implicants in the forward pass. In the reverse pass eliminate redundant prime implicants as in Prasad [6] .

Example 2: Consider the Boolean function of Example 1. Consider the largest distant set $\left(\mathrm{m}_{5}, \mathrm{~m}_{9}, \mathrm{~m}_{12}, \mathrm{~m}_{30}\right) . \mathrm{P}_{6}$ and $\mathrm{P}_{7}$ cover the min term $\mathrm{m}_{5}$. Both of them have four literals. Choose any one of them, say, $\mathrm{P}_{7}$. Similarly choose $\mathrm{P}$ ${ }_{2}, \mathrm{P}_{8}$ and $\mathrm{P}_{4}$ to cover $\mathrm{m}_{9}, \mathrm{~m}_{12}$ and $\mathrm{m}_{30}$ respectively. This gives $\left(\mathrm{P}_{7}, \mathrm{P}_{2}, \mathrm{P}_{8}, \mathrm{P}_{4}\right)$. Total number of literals in this set is 14. Therefore every minimal set of prime implicants will have at least 14 literals according to Th.2. A minimal set of prime implicants with as few literals as we can, can be obtained as follows: Consider $\mathrm{m}_{4}$. It is covered by $\mathrm{P}_{7}$ and $\mathrm{P}_{8}$ . Both of them four literals. Choose $\mathrm{P}_{7} . \mathrm{S}_{\mathrm{m}}=\left\{\mathrm{P}_{7}\right\} . \mathrm{P}_{7}$ covers $\mathrm{m}_{5}$ also. Next consider $\mathrm{m}_{9}$. Choose $\mathrm{P}_{2}$ with three literals to cover it. This gives $\mathrm{S}_{\mathrm{m}}=\left\{\mathrm{P}_{7}, \mathrm{P}_{2}\right\}$. Next consider $\mathrm{m}_{12}$ and select $\mathrm{P}_{9}$ with four literals to cover it. $\mathrm{S}_{\mathrm{m}}=\left\{\mathrm{P}_{7}\right.$, $\left.\mathrm{P}_{2}, \mathrm{P}_{9}\right\}$. Finally consider $\mathrm{m}_{15}$ and cover it with $\mathrm{P}_{4} . \mathrm{S}_{\mathrm{m}}=$ $\left\{\mathrm{P}_{7}, \mathrm{P}_{2}, \mathrm{P}_{9}, \mathrm{P}_{4}\right\}$. This covers all min terms. So the forward pass[6] is over. In the reverse pass[6] none of them can be eliminated as they are not redundant. Thus this is a minimal set of prime implicants with as few literals as possible. It has 14 literals. This is also a minimal set of prime implicants with least number of literals.

\section{Least Number of Min Term Repetitions}

We are given $\mathrm{S}_{\mathrm{d}}$ the largest distant set and all prime implicants of $f$. Ignore all don't care conditions. Let $\mathrm{P}_{\mathrm{k}}$ be a prime implicant with least number of min terms covering the min term $\mathrm{m}_{\mathrm{k}}$ of $\mathrm{S}_{\mathrm{d}}$. Do this for all $\mathrm{k}=1,2, \ldots$, d. Let $\mathrm{S}_{\mathrm{pd}}=$ $\left\{\mathrm{P}_{1}, \mathrm{P}_{2}, \ldots, \mathrm{P}_{\mathrm{d}}\right\}$. Let $\mathrm{Q}_{\mathrm{r}}$ be the set of all min terms covered by the prime implicants of $S_{p d}$. Let $P^{\prime}{ }_{k}$ be a prime implicant with least number of min terms of $Q_{r}$ covering the min term $\mathrm{m}_{\mathrm{k}}$. Do this for all $\mathrm{k}=1,2, \ldots, \mathrm{d}$. Let $\mathrm{S}_{\mathrm{pd}}=\left\{\mathrm{P}^{\prime}{ }_{1}, \mathrm{P}^{\prime}{ }_{2}\right.$, ...., $\mathrm{P}_{\mathrm{d}}$ \}. Let $\mathrm{Q}_{\mathrm{r}}$ be the set of all min terms covered by the prime implicants of $S^{\prime}{ }_{p d}$. If $Q^{\prime}{ }_{r}=Q_{r}$, the process terminates. If not , $Q_{r}^{\prime}$ has less min terms than $Q_{r}$. $Q^{\prime}{ }_{r} \rightarrow Q_{r}$. Delete $\mathrm{S}_{\mathrm{pd}}{ }_{\mathrm{d}}$. Let $\mathrm{P}{ }_{\mathrm{k}}$ be the new prime implicant with least number of min terms of the new $Q_{r}$ covering the min term $m_{k} . S^{\prime}{ }_{p d}$ is the new set of prime implicants and $Q^{\prime}{ }_{r}$ is the new set of all min terms of $S_{p d}^{\prime}$. Check if $Q_{r}^{\prime}=Q_{r}$. If this is not true repeat the process till this is true. Since $Q^{\prime}{ }_{r}$ has less min terms than $Q$ $r$, this process must terminate eventually. Let $t_{d}$ be the total number of min terms of the prime implicants of $S^{\prime}{ }_{p d}$ where a min term is counted as many times as it appears. Let $t$ be the number of min terms of $f$ where each min term is counted 
only once. Let the number of min terms of $f$ not included in $\mathrm{Q}_{\mathrm{r}}$ be $\gamma$ where each min term is counted only once.

Theorem 3: Every minimal set of prime implicants of $f$ will have at least $\left(t_{d}+\gamma-t\right)$ repetitions of min terms.

Proof: Let $\mathrm{X}=\left\{\mathrm{P}_{\mathrm{x} 1}, \mathrm{P}_{\mathrm{x} 2}, \ldots, \mathrm{P}_{\mathrm{x} \mathrm{b}}\right\}$ be the smallest minimal set of prime implicants with least number of repetitions of min terms. From Th. $1 \quad b \geq d$. Among all prime implicants of $X$ covering $\mathrm{m}_{1}$, let $\mathrm{P}_{\mathrm{x}_{1}}$ have least number of min terms of $\mathrm{Q}_{\mathrm{r}}$. Similarly $\mathrm{P}_{\mathrm{x}_{2}}$ covers $\mathrm{m}_{2}$ and so on. Let $\mathrm{P}_{\mathrm{x} k \mathrm{k}}$ be that part of $\mathrm{P}_{\mathrm{x} k}$ containing min terms of $\mathrm{Q}_{\mathrm{r}}$ only for all $\mathrm{k}=1,2, \ldots, \mathrm{d}$. Since min terms of $S_{d}$ are not close, a prime implicant of $X$ can cover at most one min term of $S_{d}$. Let $|P|$ denote the number of min terms of $\mathrm{P}$. Then $\left|\mathrm{P}_{\mathrm{x}_{\mathrm{k}}}\right| \geq\left|\mathrm{P}_{\mathrm{xk}}\right|$. Both $\mathrm{P}_{\mathrm{k}}$ and $\mathrm{P}_{\mathrm{xk}}$ cover $\mathrm{m}_{\mathrm{k}}$. But $\mathrm{P}_{\mathrm{k}}$, has least number of min terms of $\mathrm{Q}_{\mathrm{r}}$. So $\left|\mathrm{P}_{\mathrm{xk}}\right| \geq\left|\mathrm{P}_{\mathrm{k}}\right|$ $+\left|\mathrm{P}_{\mathrm{x}_{2}}\right|+\ldots \ldots+\left|\mathrm{P}_{\mathrm{xa}}\right| \geq\left|\mathrm{P}^{\prime}{ }_{1}\right|+\left|\mathrm{P}_{2}\right|+\ldots \ldots+\left|\mathrm{P}_{\mathrm{d}}\right|$ $=\mathrm{t}_{\mathrm{d}}$ and $\left|\mathrm{P}_{\mathrm{x}_{1}}\right|+\left|\mathrm{P}_{\mathrm{x}_{2}}\right|+\ldots \ldots+\left|\mathrm{P}_{\mathrm{xa}}\right|+\mid \mathrm{P}_{\mathrm{x} \mathrm{a}}+$ $1|+\ldots .+| P_{x b} \mid \geq t_{d}+\gamma$. But X covers all min terms of $f$ . Therefore at least $\left(t_{d}+\gamma-t\right)$ number of min terms must repeat in $\mathrm{X}$. This proves the theorem.

A minimal set of prime implicants with as few min term repetitions as possible can be obtained as follows: Cover each min term not covered so far by choosing a prime implicant with least number of min terms. Do this to cover all min terms and obtain an ordered set of prime implicants in the forward pass. Eliminate redundant prime implicants in the reverse pass as in Prasad [6].

Example 3: Consider the largest distant set of min terms $\left(\mathrm{m}_{5}\right.$ $, \mathrm{m}_{11}, \mathrm{~m}_{12}, \mathrm{~m}_{30}$ ) of the Boolean function of Example.1. $\mathrm{P}_{6}$ and $\mathrm{P}_{7}$ cover $\mathrm{m}_{5} . \mathrm{P}_{6}$ has fewer min terms than $\mathrm{P}_{7}$. So select $\mathrm{P}_{6}$ to cover $\mathrm{m}_{5}$. Similarly choose $\mathrm{P}_{2}, \mathrm{P}_{9}$ and $\mathrm{P}_{5}$ to cover $\mathrm{m}_{11}, \mathrm{~m}_{12}$ and $\mathrm{m}_{30}$ respectively. The min term $\mathrm{m}_{27}$ lies in $\mathrm{P}_{2}$ and $\mathrm{P}_{5}$. Other min terms covered by $\left(\mathrm{P}_{6}, \mathrm{P}_{2}, \mathrm{P}_{9}, \mathrm{P}_{5}\right)$ do not repeat. Therefore at least one min term will repeat in every minimal set of prime implicants of $f$ according to Th.3. $\left(\mathrm{P}_{2}\right.$, $\left.\mathrm{P}_{4}, \mathrm{P}_{7}, \mathrm{P}_{9}\right)$ is the smallest minimal set. $\mathrm{m}_{14}$ repeats in this set. i.e, there is at least one repetition. This shows that the smallest set of prime implicants can have least number of min term repetitions also.

\section{STATISTIC HAZARD FREE CIRCUITS}

It is well known that logic circuits can suffer from static hazards $[1,2]$. Realization of a minimal set of prime implicants covering min terms of $f$ can have static one hazards. A circuit realized from prime implicants will be free of static one hazards if every dyad of the function lies in at least one prime implicant of the minimal set [1]. Thus we need a minimal set of prime implicants covering all dyads. We can determine the least number of prime implicants required in such a minimal set as follows:

Given the min terms of $f$ obtain all dyads. Min terms of don't care conditions do not appear in these dyads. Let $\mathrm{U}=$ $\left\{d_{1}, d_{2}, \ldots, d_{y}\right\}$ be the universal set of dyads. If no prime implicant covers a dyad, it is itself a prime implicant. Therefore every dyad is covered by at least one prime implicant. A pair of dyads $d_{j}$ and $d_{k}$ is said to be close if there is a prime implicant covering both of them. Otherwise they are distant. Using this definition obtain the largest distant set $S_{y}$ of dyads. This is a subset of $U$ such that no two dyads are close. Let $\mathrm{S}_{\mathrm{y}}=\left\{\mathrm{d}^{\prime}{ }_{1}, \mathrm{~d}_{2}, \ldots, \mathrm{d}_{\alpha}\right\}$. Every minimal set of prime implicants that covers all elements of $U$ covers all elements of $\mathrm{S}_{\mathrm{y}}$ also. But no two elements of $\mathrm{S}_{\mathrm{y}}$ are covered by a single prime implicant. Therefore we need $\alpha$ number of prime implicants to cover $S_{y}$. But this set of prime implicants may or may cover all dyads. Further it may not cover all min terms also. This proves the following theorem.

Theorem 4: Every minimal set of prime implicants of $f$ free of static one hazards and covering all min terms of $f$ will have at least $\alpha$ number of prime implicants.

A minimal set of prime implicants with as few prime implicants as possible and covering all dyads and min terms can be obtained as follows: In the forward pass let $\mathrm{P}_{1}$ be a prime implicant covering a dyad $\mathrm{d}_{1}$ and containing largest number of dyads. Select $\mathrm{P}_{1}$ to cover $\mathrm{d}_{1}$. Ignore all dyads covered by $P_{1}$. Let $d_{2}$ be another dyad not covered so far. Let $P_{2}$ be another prime implicant containing largest number of uncovered dyads and covering $\mathrm{d}_{2}$. Select $\mathrm{P}_{2}$ to cover $\mathrm{d}_{2}$. In this way cover all dyads. This gives an ordered set of prime implicants. In the reverse pass eliminate redundant prime implicants, if any, as in Prasad [6]. Let $S_{m}$ be the resulting minimal set of prime implicants. If there are any min terms not lying in dyads, they are isolated or form prime implicants with don't cares. Include their prime implicants in $\mathrm{S}_{\mathrm{m}}$. This gives a minimal set of prime implicants covering all dyads and min terms. Further we tried to do this with as few prime implicants as possible.

Example 4: Consider the Boolean function of Example 1. Dyads are $\mathrm{d}_{1}:\left(\mathrm{m}_{4}, \mathrm{~m}_{5}\right), \mathrm{d}_{2}:\left(\mathrm{m}_{4}, \mathrm{~m}_{12}\right), \mathrm{d}_{3}:\left(\mathrm{m}_{9}, \mathrm{~m}_{11}\right), \mathrm{d}_{4}:\left(\mathrm{m}_{12}\right.$, $\left.\mathrm{m}_{14}\right), \mathrm{d}_{5}:\left(\mathrm{m}_{11}, \mathrm{~m}_{15}\right), \mathrm{d}_{6}:\left(\mathrm{m}_{11}, \mathrm{~m}_{27}\right), \mathrm{d}_{7}:\left(\mathrm{m}_{14}, \mathrm{~m}_{15}\right), \mathrm{d}_{8}:\left(\mathrm{m}_{14}\right.$, $\mathrm{m}_{30}$ ). Every min term lies in at least one dyad. Therefore if we cover dyads all min terms are also covered. Close dyad pairs are $\left(d_{3}, d_{6}\right),\left(d_{5}, d_{6}\right)$ and $\left(d_{7}, d_{8}\right)$. All other dyad pairs are distant pairs. $U=\left\{d_{1}, d_{2}, d_{3}, d_{4}, d_{5}, d_{6}, d_{7}, d_{8}\right\}$. Close dyad pair $\left(d_{3}, d_{6}\right)$ is destroyed if $U$ is divided into two sets. One of them does not contain $d_{3}$ while the other does not contain $d_{6}$. Delete one of $\left(d_{5}, d_{6}\right)$ from these sets in all possible ways . Later do the same for $\left(\mathrm{d}_{7}, \mathrm{~d}_{8}\right) .\left(\mathrm{d}_{1}, \mathrm{~d}_{2}, \mathrm{~d}_{3}, \mathrm{~d}_{4}, \mathrm{~d}_{5}, \mathrm{~d}_{7}\right),\left(\mathrm{d}_{1}, \mathrm{~d}_{2}, \mathrm{~d}_{3}, \mathrm{~d}\right.$ $\left.4, \mathrm{~d}_{5}, \mathrm{~d}_{8}\right)$ are the largest distant sets of dyads that result from this method. There are six dyads in each of these sets. Therefore every minimal set of prime implicants not having static one hazards requires at least six prime implicants from Th.4.

Consider the largest distant set $\left(\mathrm{d}_{1}, \mathrm{~d}_{2}, \mathrm{~d}_{3}, \mathrm{~d}_{4}, \mathrm{~d}_{5}, \mathrm{~d}_{7}\right) . \mathrm{P}_{1}, \mathrm{P}_{5}$ and $\mathrm{P}_{6}$ do not cover any dyads. Therefore they are redundant for static one hazard free circuits. The dyads covered by the remaining prime implicants are $\mathrm{P}_{2}:\left(\mathrm{d}_{3}, \mathrm{~d}_{6}\right), \mathrm{P}_{4}:\left(\mathrm{d}_{7}, \mathrm{~d}_{8}\right), \mathrm{P}_{7}$ : $\left(\mathrm{d}_{1}\right), \mathrm{P}_{8}:\left(\mathrm{d}_{2}\right)$ and $\mathrm{P}_{9}:\left(\mathrm{d}_{4}\right)$. Take $\mathrm{P}_{7}$ to cover $\mathrm{d}_{1}$. Similarly $\mathrm{P}_{8}, \mathrm{P}_{2}, \mathrm{P}_{9}, \mathrm{P}_{3}$ and $\mathrm{P}_{4}$ are taken to cover $\mathrm{d}_{2}, \mathrm{~d}_{3}, \mathrm{~d}_{4}, \mathrm{~d}_{5}$ and $\mathrm{d}_{7}$ respectively. This gives $\left(\mathrm{P}_{7}, \mathrm{P}_{8}, \mathrm{P}_{2}, \mathrm{P}_{9}, \mathrm{P}_{3}, \mathrm{P}_{4}\right)$. This covers all dyads and all min terms. Therefore this is the smallest set of prime implicants that does not have static one hazards. Note that dyad $d_{6}$ is covered more than once. i.e., every minimal set will have at least one dyad repetition. 
Remarks :

1. Given $\mathrm{S}_{\mathrm{y}}$, let $\mathrm{P}_{\mathrm{k}}$ be a prime implicant with least number of literals covering the dyad $\mathrm{d}^{\prime}{ }_{\mathrm{k}}$ for all $\mathrm{k}=1,2, \ldots, \alpha$. Then $\mathrm{S}$ $\mathrm{yd}_{\mathrm{d}}=\left(\mathrm{P}_{1}, \mathrm{P}_{2}, \ldots, \mathrm{P}_{\alpha}\right)$ is a set of prime implicants covering all dyads of $\mathrm{S}_{\mathrm{y}}$. Let $l$ be the total number of literals in $\mathrm{S}_{\mathrm{yd}}$. Then any minimal set of prime implicants of $f$ free of static one hazards will have at least $l$ literals. The proof is similar to that of Th.2.

2. Given $S_{y}$, let $P_{k}$ be a prime implicant with least number of min terms covering the dyad $\mathrm{d}^{\prime}{ }_{\mathrm{k}}$ for all $\mathrm{k}=1,2, \ldots, \alpha$. Then $\mathrm{S}_{\mathrm{yd}}=\left(\mathrm{P}_{1}, \mathrm{P}_{2}, \ldots, \mathrm{P}_{\alpha}\right)$ is a set of prime implicants covering all dyads of $\mathrm{S}_{\mathrm{y}}$. Proceeding as in Section $3 \mathrm{C}$ we can state a theorem similar to Th.3. This gives the least number of min term repetitions any static one hazard free minimal set of prime implicants will have. In this argument if $\mathrm{P}_{\mathrm{k}}$ is a prime implicant with least number of dyads then we get a lower bound on dyad repetitions.

3.If we apply Th.4 to the prime implicants of $\bar{f}$, we get the least number of prime implicates of a static zero hazard free circuit. Similarly Remarks 6 and 7 can also be stated for static zero hazard free circuits.

4.Similar to a minimal min term set, one can define a minimal dyad set. A SOP of $f$ is a minimal dyad set if (i) it covers all dyads of the function (ii) a subset of product terms does not cover all dyads (iii) no dyad is redundant. A realization of a minimal dyad set will be free of static one hazards and consumes less power.

\section{CONCLUSIONS}

Techniques are presented to derive measures like least number of (i) prime implicants (ii) literals (iii) min term repetitions and (iv) prime implicants for hazard free circuits. They are useful to assess the quality of a minimal set .The new approach tends to give smallest set of prime implicants satisfying various requirements. Minimal min term set discussed in the paper is particularly important for low power digital applications. These techniques are useful to answer similar questions for any set covering problem including some problems in graph theory.

\section{REFERENCES}

[1] D. D. Givone, "Digital Principles and Design", International Edition, Mc Graw-Hill, New York, 2003

[2] Z. Kohavi and N. K. Jha,"Switching and Finite Automata Theory", Cambridge University Press, New York, 2010, www.ambridge.org/ 9780521857482

[3] A. B. Marcovitz, "Introduction to logic design", International Edition, Mc Graw - Hill, New York, 2002.

[4] S. Minato, "Fast generation of prime irredundant covers from Binary decision Diagrams", IEICE Trans. Fundamentals, Vol. E 76 - A, No.6, pp.967-973, June 1993

[5] V. C. Prasad, "Simplification of incompletely specified sequential machines", Journal of Electrical Engineering and Electronic Technology (JEET) , Vol.4, doi:10.4172/2325-9833, 1000114, Sept.,2015

[6] V.C. Prasad, "Efficient minimization of Boolean functions", International Journal Of Electrical Engineering Education, Vol. 45/4, pp.321-327, Oct. 2008 